\title{
MicroRNA-29a induces resistance to gemcitabine through the Wnt/ק-catenin signaling pathway in pancreatic cancer cells
}

\author{
HIROAKI NAGANO, YOSHITO TOMIMARU, HIDETOSHI EGUCHI, NAOKI HAMA, HIROSHI WADA, \\ KOICHI KAWAMOTO, SHOGO KOBAYASHI, MASAKI MORI and YUICHIRO DOKI
}

Department of Surgery, Graduate School of Medicine, Osaka University, Suita, Osaka 565-0871, Japan

Received April 13, 2013; Accepted May 30, 2013

DOI: $10.3892 /$ ijo.2013.2037

\begin{abstract}
Although we studied previously the mechanisms of resistance of pancreatic cancer cells to gemcitabine (GEM), prediction of the response to GEM remains unsatisfactory. The aim of this study was to investigate the relationship between miR-29a expression and the response to GEM in pancreatic cancer cells. Changes in the growth-inhibitory effect of pancreatic cancer cells (MIAPaCa-2, PSN-1, BxPC-3 and Panc-1) to GEM were examined after overexpression or suppression of miR-29a. We also examined the effect of miR-29a on the Wnt/ $\beta$-catenin signaling pathway and investigated whether the altered growth-inhibitory effect by miR-29a suppression was weakened after the addition of Wnt $3 \mathrm{a}$, a Wnt/ $\beta$-catenin signaling activator. MIAPaCa- 2 and PSN-1 cells transfected with anti-miR-29a showed significantly lower resistance to GEM. In the anti-miR-29a-transfected cells, GEM induced significantly larger numbers of apoptotic cells and $\mathrm{S}$ phase accumulation compared to control cells, demonstrated by Annexin V assay and flow cytometric analysis of the cell cycle, respectively. The transfected cells showed overexpression of putative target molecules including Dkk1, Kremen2 and sFRP2 and lower activation of the Wnt/ $\beta$-catenin signaling pathway. The addition of Wnt3a weakened the augmented growth-inhibitory effect of anti-miR-29a transfection. Our findings suggest that miR-29a expression correlates significantly with the growth-inhibitory effect of GEM and that activation of the Wnt $\beta$-catenin signaling pathway mediated the miR-29a-induced resistance to GEM in pancreatic cancer cell lines.
\end{abstract}

\section{Introduction}

Pancreatic cancer is one of the most common malignancies worldwide (1). The prognosis of patients with pancreatic

Correspondence to: Dr Hiroaki Nagano, Department of Surgery, Graduate School of Medicine, Osaka University, 2-2 Yamadaoka E-2, Suita, Osaka 565-0871, Japan

E-mail: hnagano@gesurg.med.osaka-u.ac.jp

Key words: pancreatic cancer, gemcitabine, microRNA, miR-29a, Wnt/ $\beta$-catenin signaling pathway cancer remains poor even after curative resection and more than half of patients develop tumor recurrence at distant or local sites, with an estimated 5-year survival rate of only $20 \%$ (2-4). Chemotherapy plays an important role in the treatment of pancreatic cancer. Gemcitabine (GEM), a cell cycle specific inhibitor of DNA synthesis and a ribonucleotide reductase, has become the gold standard chemotherapeutic agent for pancreatic cancer $(5,6)$. However, the response rate to GEM is $<20 \%$, indicating that the outcome remains unsatisfactory (5). We reported that the expression of ribonucleotide reductase M1 subunit (RRM1) was significantly associated with the response to GEM in pancreatic cancer cell lines and clinical specimens $(7,8)$. However, the clinical response to GEM based on the expression of RRM1 cannot be predicted satisfactorily. Therefore, it is necessary to find novel biological markers that can accurately predict the clinical response to GEM.

Recently, microRNA (miRNA) has emerged as a critical class of negative regulators of gene expression through modulation of the post-transcriptional activity of its multiple target mRNAs by repression of translation or direct cleavage $(9,10)$. MiRNAs control a wide array of biological processes, including cell proliferation, differentiation and apoptosis. Aberrant expression of miRNAs is widely reported in human cancers with both up- and downregulation detected in cancer cells compared with their normal counterparts $(11,12)$. Employing gene manipulation protocols, the present study was designed to identify the miRNA linked to the response of pancreatic cancer cells to GEM through the modulation of Wnt/ $\beta$-catenin signaling pathway. The results showed a significant relationship between miR-29a and response to GEM in pancreatic cancer cells. Additional experiments using Wnt3a, a Wnt/ $\beta$-catenin signaling activator, demonstrated that the miR-29a-induced resistance to GEM correlated significantly with the activation of $\mathrm{Wnt} / \beta$-catenin signaling in pancreatic cancer cell lines.

\section{Materials and methods}

Pancreatic cancer cell line and clinical samples. Four human pancreatic carcinoma cell lines (MIAPaCa-2, PSN-1, BxPC-3 and Panc-1) were used in the present study (8). MIAPaCa-2 and PSN-1 cell lines were obtained from the Japanese Collection of Research Bioresources (JCRB, Tokyo, Japan). BxPC-3 and Panc- 1 cell lines were obtained from the American Type 
Culture Collection (ATCC, Rockville, MD, USA). These cells were maintained in Dulbecco's modified Eagle's medium supplemented with $10 \%$ fetal bovine serum, $100 \mathrm{U} / \mathrm{ml}$ penicillin and $100 \mathrm{mg} / \mathrm{ml}$ streptomycin at $37^{\circ} \mathrm{C}$ in a humidified incubator under $5 \% \mathrm{CO}_{2}-95 \%$ air.

Drugs and reagents. GEM was purchased from Eli Lilly and Co. (Indianapolis, IN, USA). Polyclonal rabbit anti-human DKK1 antibody (Cell Signaling Technology, Beverly, MA, USA), polyclonal rabbit anti-human sFRP2 antibody (Abcam Inc., Cambridge, MA, USA), polyclonal mouse anti-human Kremen2 antibody (Abcam Inc.) and polyclonal rabbit antihuman $\beta$-actin (Sigma-Aldrich Co., St. Louis, MO, USA) were used for western blot analysis. Recombinant human Wnt3a (R\&D Systems, Minneapolis, MN, USA) was used as a Wnt/ $\beta$-catenin signaling activator. In this study, Wnt3a was used at $50 \mathrm{ng} / \mathrm{ml}$ based on the protocol described in a previous study (13).

Transfection. Antisense miR-29a inhibitor (anti-miR-29a), miR-29a precursor (pre-miR-29a) and their negative control oligonucleotides were obtained from Ambion Inc. (Austin, TX, USA). These were used to transfect pancreatic cancer cells by using siPORT NeoFx (Ambion Inc.) according to the instructions provided by the manufacturer. The transected cells were resuspended and cultured in regular culture medium for 24-72 $\mathrm{h}$ before analysis.

RNA extraction. Total RNA and miRNA fractions were isolated from tissue samples and cell lines by TRIzol agent (Invitrogen, Carlsbad, CA, USA) and the quality of the RNA was assessed with a NanoDrop ND-1000 spectrophotometer (NanoDrop Technologies, Wilmington, DE, USA) at 260 and $280 \mathrm{~nm}(\mathrm{~A} 260 / 280)$.

Real-time quantitative reverse transcription-polymerase chain reaction for miRNA expression. Reverse transcription reaction and real-time quantitative reverse transcriptionpolymerase chain reaction (qRT-PCR) were performed using TaqMan human miRNA assay kit (Applied Biosystems, Foster City, CA, USA) according to the instructions supplied by the manufacturer. The expression of the target miRNA was normalized relative to that of the internal control; RNU48. Data were analyzed according to the comparative $\mathrm{Ct}$ method (14).

Real-time $q R T$-PCR for $m R N A$ expression. RT reaction was performed with SuperScript II (Invitrogen) based on the protocol provided by the manufacturer, followed by qRT-PCR The expression of the target gene was normalized relative to the expression of porphobilinogen deaminase (PBGD), which was used as an internal control. The designed PCR primers were as follows; AXIN2 forward primer, 5'-GGTGTTTGAGG AGATCTGGG-3'; AXIN2 reverse primer, 5'-TGCTCACAGC CAAGACAGTT-3'; CCND1 forward primer, 5'-AAGGCCTG AACCTGAGGAG-3'; $C C N D 1$ reverse primer, 5'-CTTGACTC CAGCAGGGCTT-3'; $M Y C$ forward primer, 5'-AAGAGGAC TTGTTGCGGAAA-3'; MYC reverse primer, 5'-CTCAGCCA AGGTTGTGAGGT-3'; TACSTDI forward primer, 5'-TCCAG AAAGAAGAGAATGGCA-3'; TACSTD1 reverse primer, 5'-AAAGATGTCTTCGTCCCACG-3'; TCF3 forward primer,
5'-ATCTGTGTCCCATGTCCCAG-3'; TCF3 reverse primer, 5'-CCAGGGTAGGAGACTTGCAG-3'; and $P B G D$ forward primer, 5'-TGTCTGGTAACGGCAATGCGGCTGCAAC-3'; $P B G D$ reverse primer, 5'-TCAATGTTGCCACCACACTGT CCGTCT-3'.

Western blot analysis. Cells grown to semiconfluence were lysed in RIPA buffer [25 mM Tris (pH 7.5), $50 \mathrm{mM} \mathrm{NaCl}$, $0.5 \%$ sodium deoxycholate, $2 \%$ Nonidet P- $40,0.2 \%$ sodium dodecyl sulfate, $1 \mathrm{mM}$ phenylmethylsulphonyl fluoride and $500 \mathrm{KIE} / \mathrm{ml}$ Trasylol, proteinase inhibitor (Bayer, Leverkusen, Germany)]. Western blot analysis was carried out as described previously $(15,16)$.

Growth-inhibitory assay. Inhibition of cell growth in the presence of chemotherapeutic agents was assessed by the 3-(4-,5-dimethylthiazol-2-yl)-2,5-diphenyl tetrazolium bromide (MTT) (Sigma-Aldrich Co.) assay as described previously $(15,17)$. Briefly, the cells were incubated for $72 \mathrm{~h}$ under various concentrations of GEM. After re-incubation for $4 \mathrm{~h}$ in MTT solution, acid-isopropanol was added to dissolve the resultant formazan crystals. The absorbance of the plate was measured in a microplate reader at a wavelength of $570 \mathrm{~nm}$ with a $650-\mathrm{nm}$ reference and the results were expressed as the percentage of absorbance relative to untreated controls.

Annexin $V$ assay. The binding of Annexin $\mathrm{V}$ was used as a sensitive method for measuring apoptosis, as described previously (15). Twenty-four hours after treatment, cells were stained with Annexin V-FITC and propidium iodide (PI) (BioVision Research Products, Mountain View, CA, USA) and analyzed on a FACSCalibur (BD Biosciences, Franklin Lakes, NJ, USA). For the assessment of apoptosis, Annexin V-positive and PI-negative cells and Annexin V-positive and PI-positive cells were considered as early apoptotic cells and late apoptotic cells, respectively.

Cell cycle analysis. Cell cycle analysis was performed based on flow cytometric analysis, as described previously $(16,17)$. Briefly, PI and RNase (Sigma-Aldrich Co.) were added and data were acquired on the FACSCalibur (BD Biosciences). The cell cycle was analyzed using ModFIT software (BD Biosciences).

Luciferase reporter assay. To evaluate the activity of the Wnt/ $\beta$-catenin signaling pathway, TCF/LEF transcriptional activity was examined. For the examination, the reporter assay kit (SA Biosciences, Frederick, MD, USA) was used according to the instructions provided by the manufacturer. In brief, cells were transiently transfected with the transcription factor-responsive reporter or negative control by the Lipofectamine 2000 reagent (Invitrogen). After the transfection, the cells were transfected with anti-miR-29a or its negative control oligonucleotide. After $48 \mathrm{~h}$, luciferase activity was measured with the Dual-Luciferase Assay System (Promega, Madison, WI, USA) using luminometer, Lumat LB9507 (Berthold Technologies, Calmbacher, Germany). The Firefly luciferase activity, indicating TCF-dependent transcription, was normalized to the Renilla luciferase activity as an internal control to obtain the relative luciferase activity. 


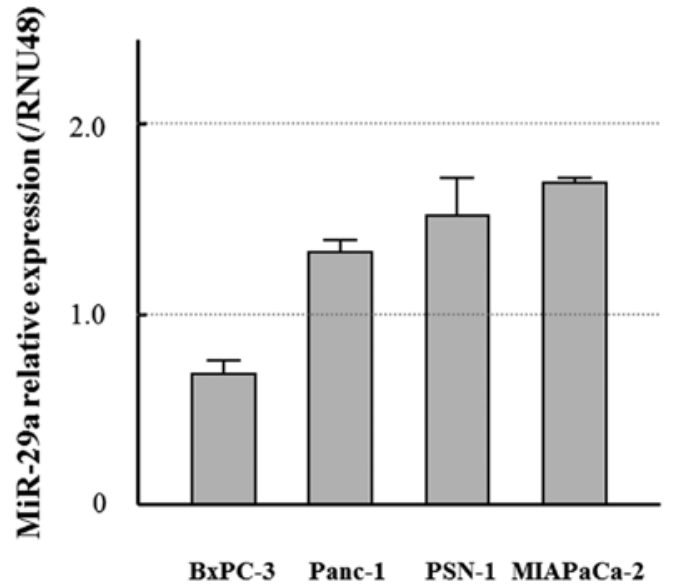

Figure 1. The expression level of miR-29a in four human pancreatic carcinoma cell lines including MIAPaCa-2, PSN-1, BxPC-3 and Panc-1. The miR-29a expression was normalized by the average expression of BxPC-3 with the lowest expression of miR-29a in the pancreatic cancer cell lines. Data are mean \pm SD

Statistical analysis. Data were expressed as mean \pm SD. Clinicopathological parameters were compared using the $\chi^{2}$ test and continuous variables were compared using the Student's t-test. A p $<0.05$ denoted the presence of a statistically significant difference. Statistical analysis was performed using the StatView software (SAS Institute Inc., Cary, NC, USA).

\section{Results}

Transfection of anti-miR-29a reduces resistance to GEM. All the four cell lines used in the present study expressed miR-29a though the level varied among the cells (Fig. 1). The relative expression of miR-29a was significantly lower in the transfected cells by qRT-PCR (Fig. 2A). To evaluate the effect of miR-29a on the response to GEM, we transfected antimiR-29a in MIAPaCa-2 and PSN-1 cells, which showed higher miR-29a expression levels than the other cell lines. The MTT assay showed that cells transfected with anti-miR-29a were significantly less resistant to GEM compared to control cells (Fig. 2B). Next, we evaluated the extent of apoptosis of these cells at $24 \mathrm{~h}$ after treatment with GEM (MIAPaCa-2; $40 \mathrm{ng}$ / $\mathrm{ml}, \mathrm{PSN}-1 ; 2 \mathrm{ng} / \mathrm{ml}$ ) by the Annexin V assay. The percentages of early and late apoptotic cells were significantly higher in the two cancer cell lines transfected with anti-miR-29a than in the control cells (Fig. 2C).

We also examined the influence of miR-29a on the cell cycle in MIAPaCa-2 and PSN-1. The distribution of cells in $\mathrm{G}_{0} / \mathrm{G}_{1}$ phase, $S$ phase and $\mathrm{G}_{2} / \mathrm{M}$ phase was similar between the miR-29a-suppressed cells and the control cells in the absence of GEM (Fig. 2D). However, $24 \mathrm{~h}$ after GEM treatment (MIAPaCa-2; $20 \mathrm{ng} / \mathrm{ml}, \mathrm{PSN}-1 ; 1 \mathrm{ng} / \mathrm{ml}$ ), the percentage of cells at the $\mathrm{S}$ phase among the miR-29a-suppressed cells was higher than the control cells (Fig. 2D).

MiR-29a activates Wnt/ $\beta$-catenin signaling pathway. We examined next the expression levels of these molecules in MIAPaCa-2 and PSN-1. Western blot analysis showed significantly higher protein expression levels of these molecules in the anti-miR-29a-transfected cells compared with the control cells (Fig. 3A). Furthermore, the luciferase reporter assay showed that TCF/LEF transcriptional activity, representing the activity of the Wnt/ $\beta$-catenin signaling pathway, was significantly lower in the miR-29a-suppressed cells than in the control cells (Fig. 3B). We also examined the expression of five $\mathrm{Wnt} / \beta$-catenin signaling targeted genes (AXIN2, CCND1, $M Y C, T A C S T D 1$ and TCF3) in anti-miR-29a-transfected MIAPaCa-2 and PSN-1 cell lines by qRT-PCR. Suppression of miR-29a significantly reduced the mRNA expression of the targeted genes (Fig. 3C). Taken together, the results suggest that miR-29a activates the $\mathrm{Wnt} / \beta$-catenin signaling pathway through the suppression of Dkk1, Kremen2 and sFRP2.

Transfection of pre-miR-29a induces resistance to GEM. To further assess the effects of miR-29a, pre-miR-29a was transfected into BxPC-3 and Panc-1, which expressed lower levels of miR-29a than the other cell lines (Fig. 1). Transfection of cells with pre-miR-29a increased miR-29a level compared to the control cells (Fig. 4A). The miR-29a-overexpressing cells were significantly more resistant to GEM than the control cells, as evident by the MTT assay (Fig. 4B).

MiR-29a-induced resistance is mediated by Wnt/ $\beta$-catenin signaling activation. Finally, we analyzed the mechanism responsible for the miR-29a-induced resistance to GEM. We focused on the Wnt/ $\beta$-catenin signaling pathway, based on the results reported by Kapinas et al (13). The addition of Wnt3a to the cultures of $\mathrm{MIAPaCa}-2$ and $\mathrm{PSN}-1$ resulted in the activation of $\mathrm{Wnt} / \beta$-catenin signal in the cell lines (Fig. 5A). Furthermore, the MTT assay showed the Wnt3a-treated cells were more resistant to GEM (MIAPaCa-2; $25 \mathrm{ng} / \mathrm{ml}, \mathrm{PSN}-1$; $1.6 \mathrm{ng} / \mathrm{ml}$, Fig. 5B). In addition, both the inactivated $\mathrm{Wnt} / \beta$ catenin signal and the augmented growth-inhibitory effect by the afore-mentioned anti-miR-29a transfection were weakened after the addition of Wnt3a (Fig. 5). These findings suggest that activation of the $\mathrm{Wnt} / \beta$-catenin signaling mediates, at least in part, the miR-29a-induced resistance to GEM.

\section{Discussion}

The present study demonstrated that the expression of miR-29a correlated significantly with the growth-inhibitory effect of GEM and that the Wnt/ $\beta$-catenin signal mediates the miR-29a-induced resistance to GEM in pancreatic cancer cell lines. Kapinas et al reported previously that miR-29a activates the $\mathrm{Wnt} / \beta$-catenin signal through direct regulation of the negative regulators of the signal, Dkk1, Kremen2 and sFRP2 (13). Other studies indicated that activation of the Wnt/ $\beta$-cateninsignaling, which is observed in $65 \%$ of pancreatic cancer cases, also plays an important role in the proliferation and differentiation of stem cells and that some chemotherapeutic drugs often induce tumor cell death, but not cancer stem cells (18-22). Moreover, the Wnt/ $\beta$-catenin signal was reported to correlate significantly with chemoresistance (16,23-25). Thus, the results of the present study are in agreement with the above previous reports.

On the other hand, the Wnt/ $\beta$-catenin signaling pathway, which plays important roles in the development of various malignancies, cell proliferation and differentiation, has been also reported to correlate with chemoresistance (23-25). In 
A

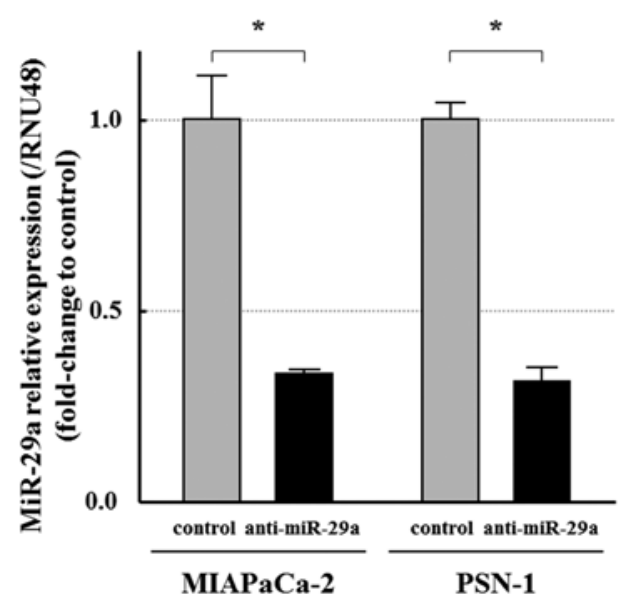

B

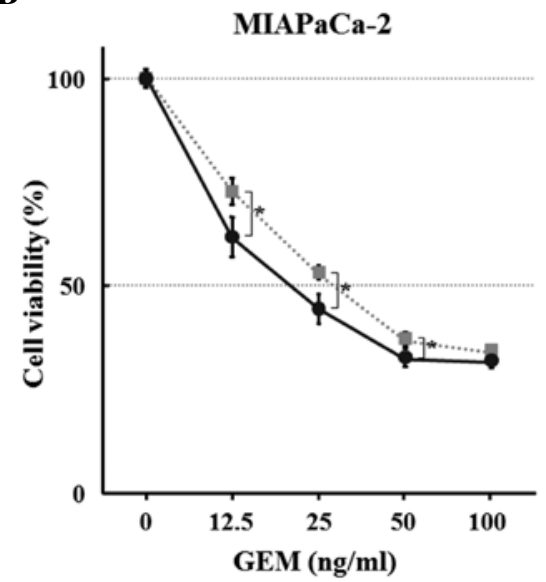

PSN-1

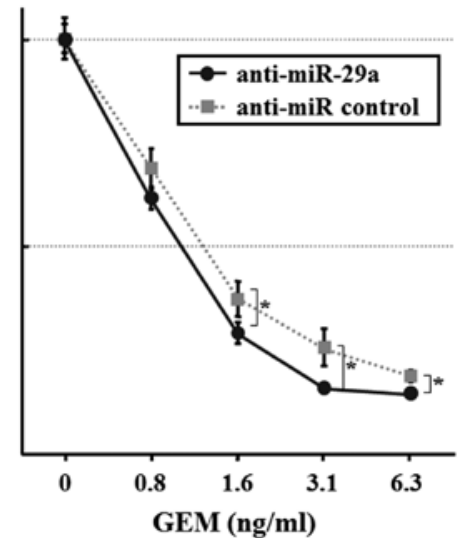

C

Annexin V (+)/PI (-)

Annexin V (+)/PI (+)

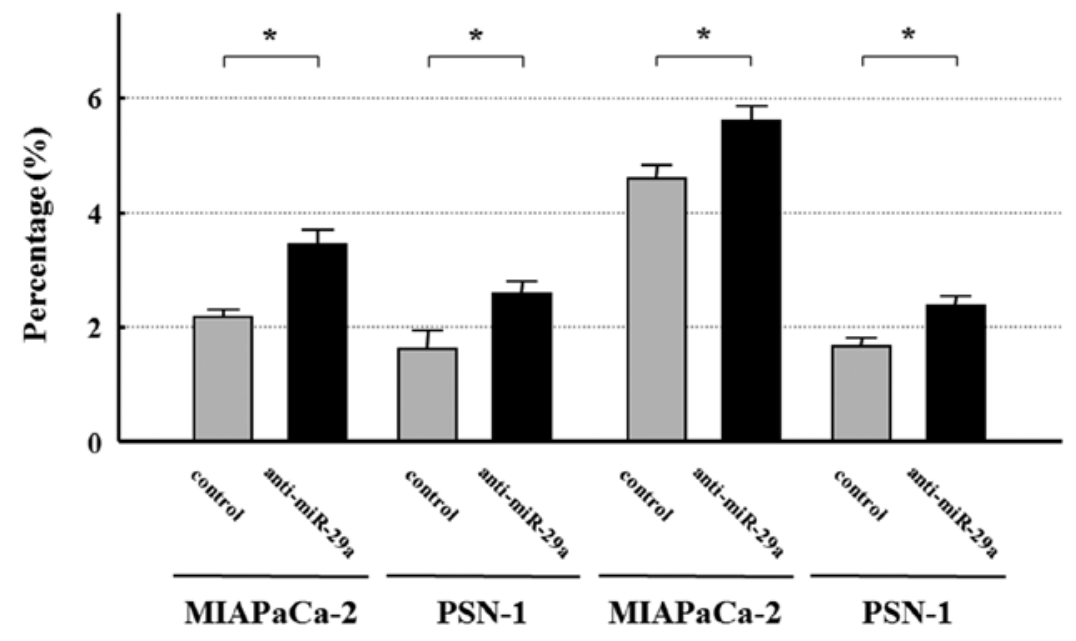

D

MIAPaCa-2

control

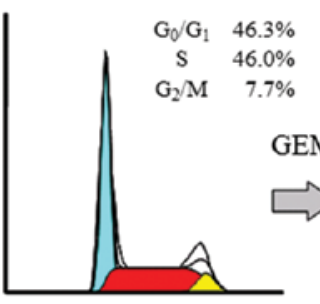

anti-miR-29a

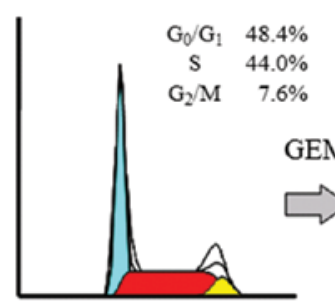

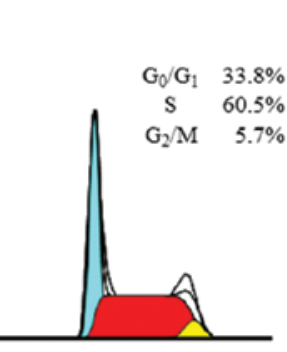

control

\section{PSN-1}

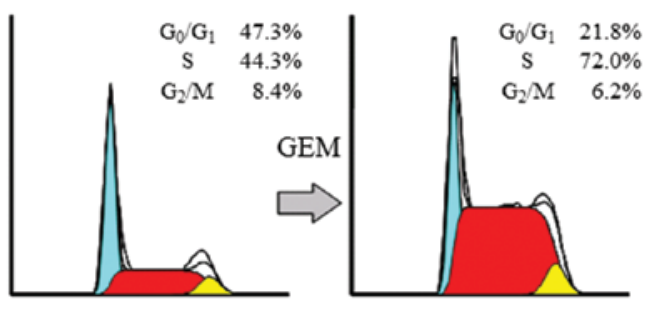

anti-miR-29a

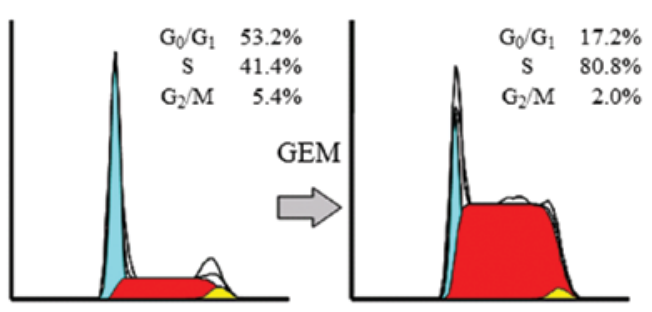

$\mathrm{G}_{0} / \mathrm{G}_{1}$ phase

$\mathrm{S}$ phase

$\mathrm{G}_{2} / \mathrm{M}$ phase

Figure 2. Transfection of anti-miR-29a into MIAPaCa-2 and PSN-1. (A) qRT-PCR showed significant suppression of miR-29a in the transfected cells compared to the control cells $(" \mathrm{p}<0.05)$. (B) MTT assay showed that the growth-inhibitory effects of GEM in the miR-29a-suppressed cells was significantly stronger than in the control cells ( $\left.{ }^{*}<0.05\right)$. (C) Annexin V assay indicated that the percentages of early apoptotic cells and late apoptotic cells induced by GEM (MIAPaCa-2; $40 \mathrm{ng} / \mathrm{ml}$, PSN-1; $2 \mathrm{ng} / \mathrm{ml}$ ) in the miR-29a-suppressed cells were significantly higher than in the control cells ("p<0.05). (D) Flow cytometric analysis of the cell cycle indicated that GEM treatment (MIAPaCa-2; $20 \mathrm{ng} / \mathrm{ml}, \mathrm{PSN}-1 ; 1 \mathrm{ng} / \mathrm{ml}$ ) resulted in accumulation of cells in the S phase among the miR-29a-suppressed cells compared to the control cells. Data are mean $\pm \mathrm{SD}$. 
A

$\frac{\text { MIAPaCa-2 }}{\text { control anti-miR-29a }}$

Dkk1

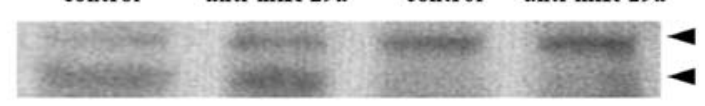

\section{Kremen2}

SFRP2

$\beta$-actin

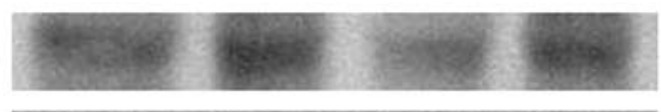

C
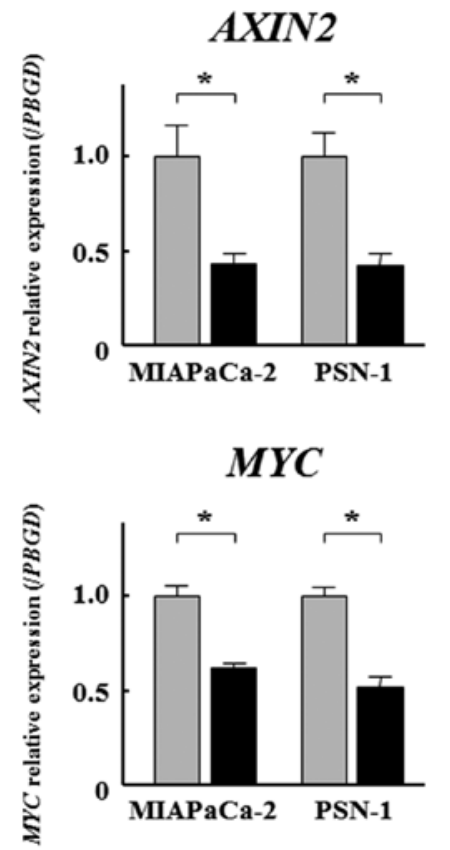

B

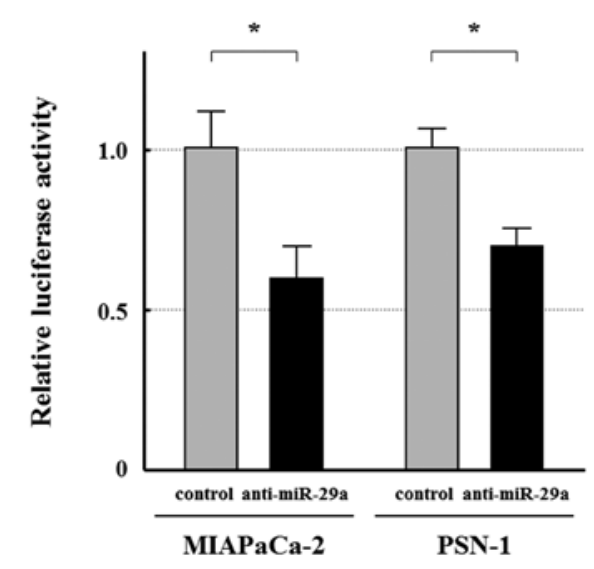

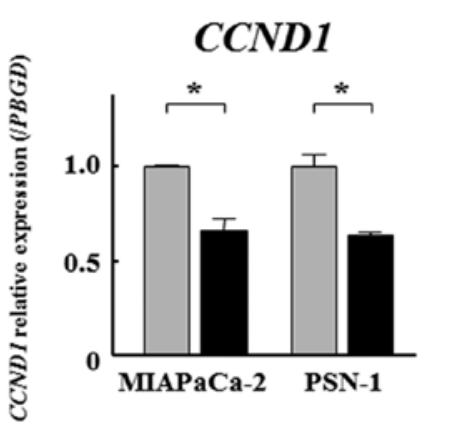
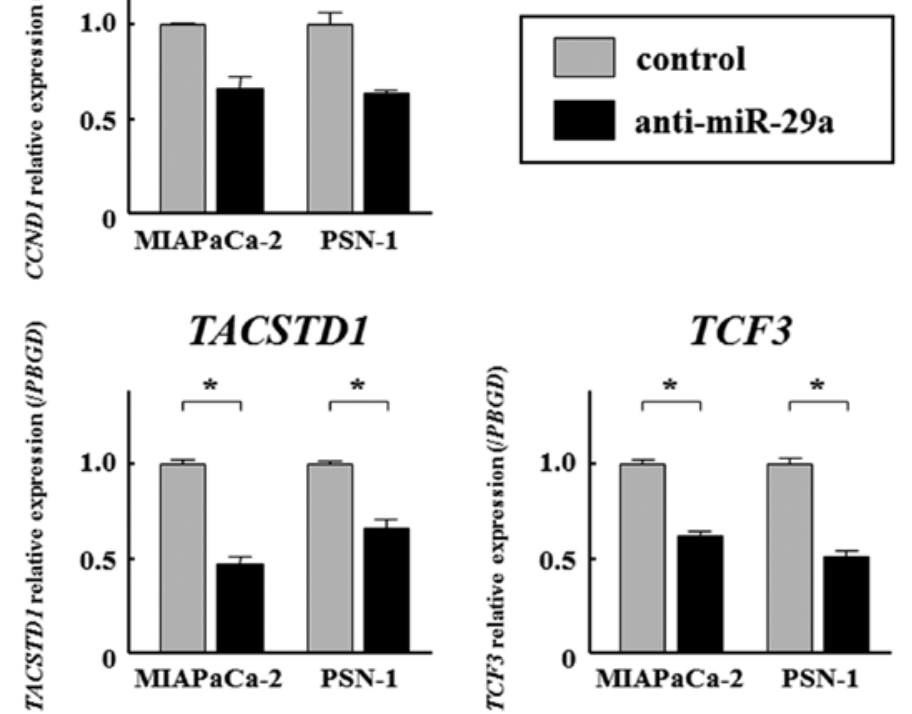

Figure 3. Evaluation of the influence of the Wnt/ß-catenin signaling pathway in MIAPaCa-2 and PSN-1. (A) Western blot analysis demonstrated significant overexpression of Dkk1, Kremen2 and sFRP2 proteins in the anti-miR-29a-transfected cells compared to the control cells. (B) Luciferase reporter assay showed that TCF/LEF transcriptional activity was significantly lower in the miR-29a-suppressed cells than in the control cells ("p<0.05). (C) qRT-PCR demonstrated significantly lower expressions levels of five Wnt/ $\beta$-catenin signaling targeted genes in the miR-29a-suppressed cells than in the control cells $($ " $<<0.05)$. Data are mean \pm SD.
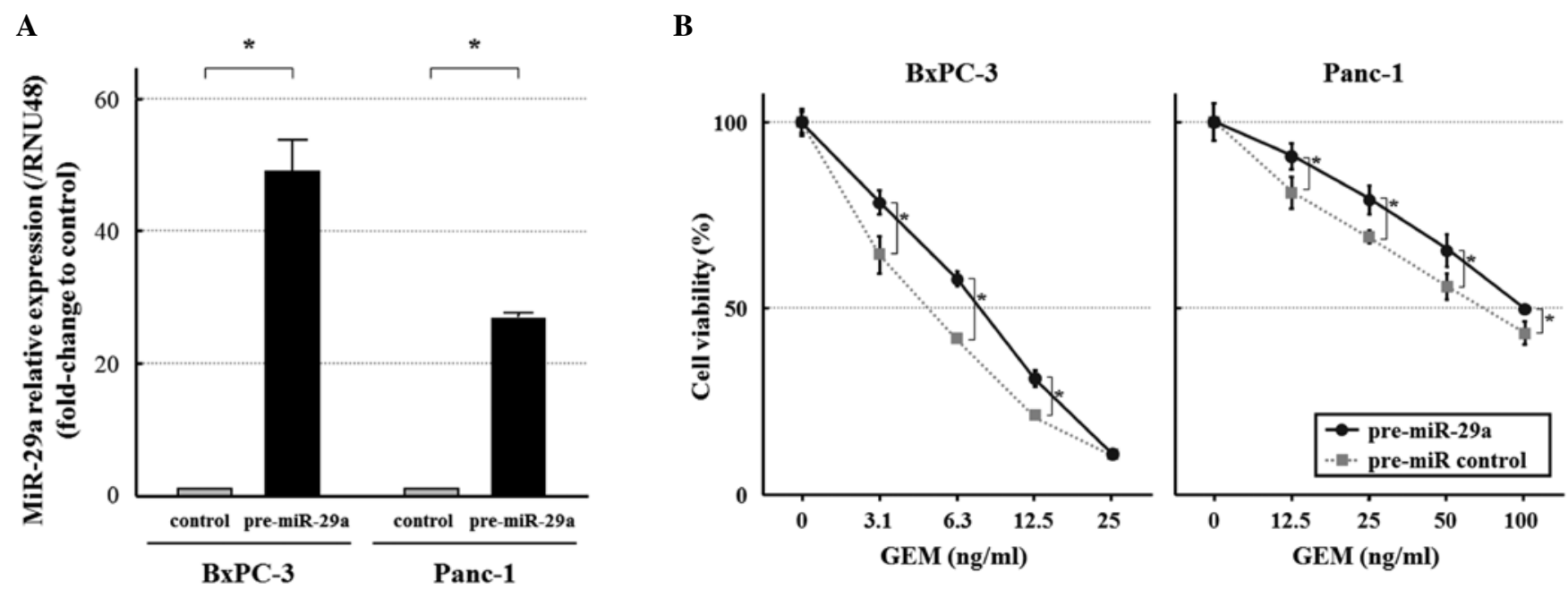

Figure 4. Effects of transfection of pre-miR-29a into BxPC-3 and Panc-1. (A) Overexpression of miR-29a in the transfected cells was confirmed by qRT-PCR ( $\mathrm{p}<0.05)$. (B) MTT assay showed that the growth-inhibitory effects of GEM in the miR-29a-overexpressed cells was significantly weaker than in the control cells $($ " $\mathrm{p}<0.05)$. Data are mean \pm SD. 
A

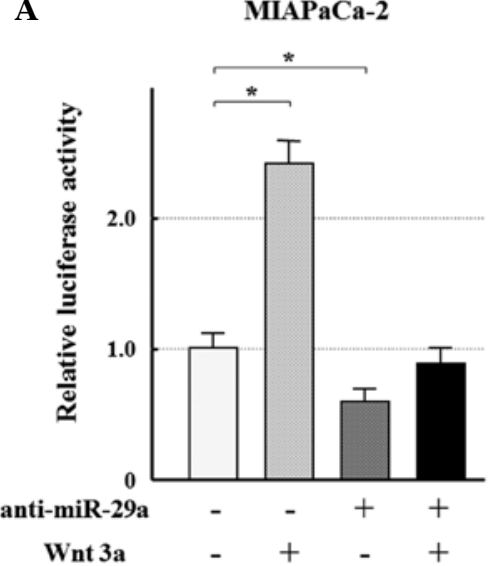

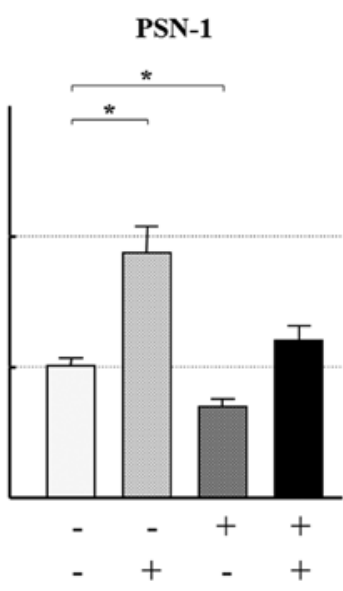

B

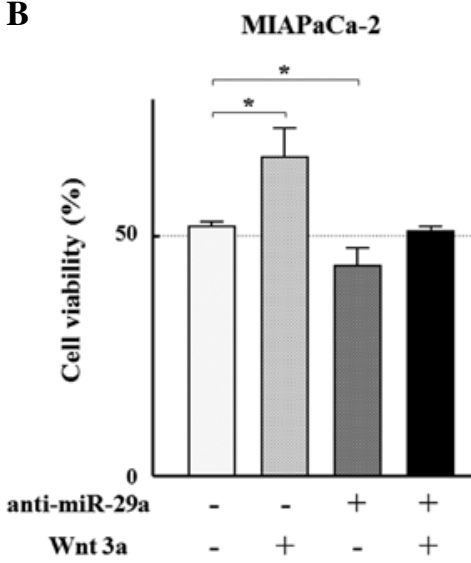

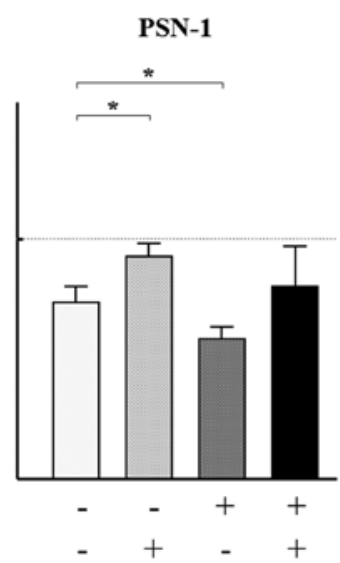

Figure 5. Changes in Wnt/ $\beta$-catenin signaling activity and growth-inhibitory effects of GEM after anti-miR-29a transfection and/or Wnt3a addition. MTT assay indicated a weaker growth-inhibitory effect for GEM (MIAPaCa-2; $25 \mathrm{ng} / \mathrm{ml}, \mathrm{PSN}-1 ; 1.6 \mathrm{ng} / \mathrm{ml}$ ) following the addition of Wnt3a at 50 ng/ml and that the strengthened growth-inhibitory effect by anti-miR-29a transfection was also weakened after the addition of Wnt3a ( $\left.{ }^{*} \mathrm{p}<0.05\right)$. Data are mean \pm SD.

fact, we reported previously the activation of the Wnt/ $\beta$-catenin signaling pathway in HCC with poor response to interferon and 5-fluorouracil therapy (16). Recently, Kapinas et al (13) reported that miR-29a activates the $\mathrm{Wnt} / \beta$-catenin signal by directly regulating Dikkopf-1 (Dkk1), Kremen2 and secreted frizzled related protein 2 (sFRP2), which are negative regulators of the signal transduction, suggesting that miR-29a induces chemoresistance to chemotherapeutic agents through the activation of the $\mathrm{Wnt} / \beta$-catenin signaling pathway.

We reported previously that RRM1 expression correlates significantly with the response to $\operatorname{GEM}(7,8)$. Therefore, in the present study, we also investigated the effects of anti-miR-29a transfection on RRM1 expression. The result showed no significant change in RRM1 expression after anti-miR-29a transfection (data not shown). Several investigators also reported that the expression of miRNAs correlates significantly with chemoresistance in several types of cancers $(15,26,27)$. For example, we reported that the expression of miR-21 is associated with resistance to interferon and 5-fluorouracil in hepatocellular carcinoma (HCC) (13), while others indicated that such expression is associated with response to GEM in pancreatic cancer and cholangiocarcinoma cells $(15,26,27)$.

Furthermore, since miRNA is associated with the response to GEM, miR-21 has been reported in some studies of pancreatic and other cancers $(15,26,27)$. Therefore, in the present study, the effects of transfection of anti-miR-29a on miR-21 expression were also examined and the results showed no significant changes in miR-21 expression (data not shown). These results suggest that the chemoresistance induced by miR-29a is different from that related to RRM1 and miR-21.

To date, evidence suggests that miR-29a acts as an oncomiRNA as well as an anti-oncomiRNA (28-31). Xiong et al (28) reported that miR-29a promotes apoptosis and represses tumorigenicity in HCC cells, while the present study showed contradictory results. The reason for this contradiction remains unresolved, but it is speculated that miR-29a can act as either an oncomiRNA or an anti-oncomiRNA, depending on the tumor circumstances, suggesting that the exact role of miR-29a in cancer is still unclear and needs to be fully investigated in the future.
Several studies have reported that miR-29a is detected in the sera of patients with ovarian and colorectal cancers, suggesting its potential use as a biomarker for cancer detection $(32,33)$. Confirmation of the present findings in larger population multicenter studies may allow the measurement of plasma levels of miR-29a to predict the clinical response to GEM in patients with pancreatic cancer.

In conclusion, the present study demonstrated a significant association between miR-29a expression and the response to GEM in pancreatic cancer cell lines by genetic manipulation experiments. The results showed that the miR-29a-induced resistance to GEM is mediated by activation of the $\mathrm{Wnt} / \beta$ catenin signaling pathway. These findings suggest that miR-29a could be potentially used as a marker for the prediction of the clinical response to GEM and serves as a potential target for therapy against pancreatic cancer.

\section{References}

1. Warshaw AL and Fernandez-del Castillo C: Pancreatic carcinoma. N Engl J Med 326: 455-465, 1992.

2. Li D, Xie K, Wolff R and Abbruzzese JL: Pancreatic cancer. Lancet 363: 1049-1057, 2004.

3. Neoptolemos JP, Stocken DD, Friess H, et al: A randomized trial of chemoradiotherapy and chemotherapy after resection of pancreatic cancer. N Engl J Med 350: 1200-1210, 2004.

4. Sener SF, Fremgen A, Menck HR and Winchester DP: Pancreatic cancer: a report of treatment and survival trends for 100,313 patients diagnosed from 1985-1995, using the National Cancer Database. J Am Coll Surg 189: 1-7, 1999.

5. Burris HA III, Moore MJ, Andersen J, et al: Improvements in survival and clinical benefit with gemcitabine as first-line therapy for patients with advanced pancreas cancer: a randomized trial. J Clin Oncol 15: 2403-2413, 1997.

6. Oettle $\mathrm{H}$, Post $\mathrm{S}$, Neuhaus $\mathrm{P}$, et al: Adjuvant chemotherapy with gemcitabine vs observation in patients undergoing curativeintent resection of pancreatic cancer: a randomized controlled trial. JAMA 297: 267-277, 2007.

7. Akita H, Zheng Z, Takeda Y, et al: Significance of RRM1 and ERCC1 expression in resectable pancreatic adenocarcinoma. Oncogene 28: 2903-2909, 2009.

8. Nakahira S, Nakamori S, Tsujie M, et al: Involvement of ribonucleotide reductase M1 subunit overexpression in gemcitabine resistance of human pancreatic cancer. Int J Cancer 120: 1355-1363, 2007.

9. Calin GA and Croce CM: MicroRNA-cancer connection: the beginning of a new tale. Cancer Res 66: 7390-7394, 2006. 
10. Bartel DP: MicroRNAs: genomics, biogenesis, mechanism and function. Cell 116: 281-297, 2004.

11. Croce $\mathrm{CM}$ and Calin GA: miRNAs, cancer and stem cell division. Cell 122: 6-7, 2005.

12. Calin GA and Croce CM: MicroRNA signatures in human cancers. Nat Rev Cancer 6: 857-866, 2006.

13. Kapinas K, Kessler C, Ricks T, Gronowicz G and Delany AM: miR-29 modulates Wnt signaling in human osteoblasts through a positive feedback loop. J Biol Chem 285: 25221-25231, 2010.

14. Schmittgen TD, Jiang J, Liu Q and Yang L: A high-throughput method to monitor the expression of microRNA precursors. Nucleic Acids Res 32: e43, 2004.

15. Tomimaru Y, Eguchi H, Nagano H, et al: MicroRNA-21 induces resistance to the anti-tumor effect of interferon- $\alpha / 5$-fluorouracil in hepatocellular carcinoma cells. Br J Cancer 103: 1617-1626, 2010.

16. Noda T, Nagano H, Takemasa I, et al: Activation of Wnt/ beta-catenin signalling pathway induces chemoresistance to interferon-alpha/5-fluorouracil combination therapy for hepatocellular carcinoma. Br J Cancer 100: 1647-1658, 2009.

17. Eguchi H, Nagano H, Yamamoto H, et al: Augmentation of antitumor activity of 5-fluorouracil by interferon alpha is associated with up-regulation of p27Kip1 in human hepatocellular carcinoma cells. Clin Cancer Res 6: 2881-2890, 2000

18. Jamieson CH, Ailles LE, Dylla SJ, et al: Granulocyte-macrophage progenitors as candidate leukemic stem cells in blast-crisis CML. N Engl J Med 351: 657-667, 2004.

19. Klaus A and Birchmeier W: Wnt signalling and its impact on development and cancer. Nat Rev Cancer 8: 387-398, 2008.

20. Pasca di Magliano M, Biankin AV, Heiser PW, et al: Common activation of canonical Wnt signaling in pancreatic adenocarcinoma. PLoS One 2: e1155, 2007.

21. Zeng G, Germinaro M, Micsenyi A, et al: Aberrant Wnt/betacatenin signaling in pancreatic adenocarcinoma. Neoplasia 8: 279-289, 2006.

22. Barker $\mathrm{N}$ and Clevers $\mathrm{H}$ : Mining the Wnt pathway for cancer therapeutics. Nat Rev Drug Discov 5: 997-1014, 2006.

23. Yang W, Yan HX, Chen L, et al: Wnt/beta-catenin signaling contributes to activation of normal and tumorigenic liver progenitor cells. Cancer Res 68: 4287-4295, 2008.
24. Su HY, Lai HC, Lin YW, et al: Epigenetic silencing of SFRP5 is related to malignant phenotype and chemoresistance of ovarian cancer through Wnt signaling pathway. Int J Cancer 127: 555-567, 2010.

25. Flahaut M, Meier R, Coulon A, et al: The Wnt receptor FZD1 mediates chemoresistance in neuroblastoma through activation of the Wnt/beta-catenin pathway. Oncogene 28: 2245-2256, 2009.

26. Giovannetti E, Funel N, Peters GJ, et al: MicroRNA-21 in pancreatic cancer: correlation with clinical outcome and pharmacologic aspects underlying its role in the modulation of gemcitabine activity. Cancer Res 70: 4528-4538, 2010.

27. Ali S, Ahmad A, Banerjee S, et al: Gemcitabine sensitivity can be induced in pancreatic cancer cells through modulation of miR-200 and miR-21 expression by curcumin or its analogue CDF. Cancer Res 70: 3606-3617, 2010.

28. Xiong Y, Fang JH, Yun JP, et al: Effects of microRNA-29 on apoptosis, tumorigenicity and prognosis of hepatocellular carcinoma. Hepatology 51: 836-845, 2010.

29. Fabbri M, Ivan M, Cimmino A, Negrini M and Calin GA: Regulatory mechanisms of microRNAs involvement in cancer. Expert Opin Biol Ther 7: 1009-1019, 2007.

30. Gebeshuber CA,Zatloukal K and Martinez J: miR-29a suppresses tristetraprolin, which is a regulator of epithelial polarity and metastasis. EMBO Rep 10: 400-405, 2009.

31. Han YC, Park CY, Bhagat G, et al: microRNA-29a induces aberrant self-renewal capacity in hematopoietic progenitors, biased myeloid development and acute myeloid leukemia. J Exp Med 207: 475-489, 2010.

32. Resnick KE, Alder H, Hagan JP, Richardson DL, Croce CM and Cohn DE: The detection of differentially expressed microRNAs from the serum of ovarian cancer patients using a novel real-time PCR platform. Gynecol Oncol 112: 55-59, 2009.

33. Huang Z, Huang D, Ni S, Peng Z, Sheng W and Du X: Plasma microRNAs are promising novel biomarkers for early detection of colorectal cancer. Int J Cancer 127: 118-126, 2010. 